\title{
Nonconvex Lifted Unbalanced Branch Flow Model: Derivation, Implementation and Experiments
}

\author{
Frederik Geth \\ CSIRO Energy \\ Newcastle NSW, Australia \\ frederik.geth@csiro.au
}

\author{
Sander Claeys \\ Geert Deconinck \\ KU Leuven and EnergyVille \\ Leuven and Genk, Belgium \\ sander.claeys@kuleuven.be
}

\begin{abstract}
This paper develops a novel nonconvex formulation of the unbalanced power flow equations. This formulation extends the lifted nonconvex 'DistFlow' a.k.a. balanced branch flow model formulation to the unbalanced case. The feasible set is characterized by linear and nonconvex quadratic equations in matrix variables. It is shown that this formulation is equivalent to previously published rank-constrained semidefinite programming formulations. The formulation is implemented and compared numerically with rectangular and polar versions of the nonlinear AC unbalanced optimal power flow problem.
\end{abstract}

Index Terms-Unbalanced Optimal Power Flow, Mathematical Optimization

\section{INTRODUCTION}

We refer to [1] for a recent in-depth review of mathematical formulations for the optimal power flow (OPF) problem. It is noted that OPF formulations are commonly developed in the complex power-voltage variable space, instead of the currentvoltage variable space common to power flow solvers. The 'DistFlow' formulation was developed by Baran and Wu [2]. The fundamental formulation is quadratic-nonconvex. Lifting and convex relaxation techniques have been applied to it, to obtain a second-order conic representation (also called the convex branch flow model, BFM). This in turn has been extended to include transformer models and line charging effects [3]. The properties of the convex BFM have been described in detail [4].

A key feature of the lifted convex BFM is that it often returns in AC-feasible solutions for radial grids. Nevertheless, both the original nonconvex and the convex variants are relaxations of the $\mathrm{AC}$ power flow equations when used to model meshed grids. The underlying issue is that the phase angle shift over branches are unaccounted for in the mathematical model. In a radial grid, voltage angles don't impact the power flow, so one can generally recover the angle values in postprocessing. In meshed grids however, the voltage angles over a loop in the mesh must also add up to $0 \bmod 2 \pi$, which alters how power flows.

Distribution grids are often radial, and therefore there has been significant interest in using these formulations when dealing with distribution grid optimization problems. Nevertheless, in distribution grids, where single-phase load and DER are common and aggregation is limited, one may observe nonnegligible amounts of voltage or current phase unbalance.
Such physical effects are not considered in the those "balanced' power flow formulations. Unbalanced power flow is the modelling framework underlying the steady state physics of three-phase power grids with nonneglegible phase unbalance. The underlying problem is posed in Kirchhoff's circuit laws, which are linear equations in current and voltage vectors for known branch impedance matrices.

The first rank-constrained semi-definite programming (SDP) formulation of the unbalanced OPF problem, and its SDP relaxation, were proposed by Dall'Anese et al. [5]. Gan et al. developed the BFM variant, and defined BFM and the bus injection model in a consistent notation [6]. We note that an extension to the lifted quadratic nonconvex BFM formulation that includes phase unbalance, so far has not been proposed in the literature. Before we start the derivation, we illustrate the nonconvex BFM formulation in the power-voltage variable space (nonlifted).

\section{A. Nonconvex Unbalanced BFM}

Fig. 1 summarizes the variables and parameters defined in the fundamental $3 \times 3$ branch model. All circuit element voltages are defined w.r.t. (local) ground voltage $U_{i, g}=0 \mathrm{~V}$. The total current in conductor $a$ of branch $l$, flow from $i$ to $j$ is $I_{l i j, a}$, the series part of it is $I_{l i j, a}^{\mathrm{s}}$, the from-side shunt part is $I_{l i j, a}^{\text {sh }}$. We now illustrate the formulation of the BFM

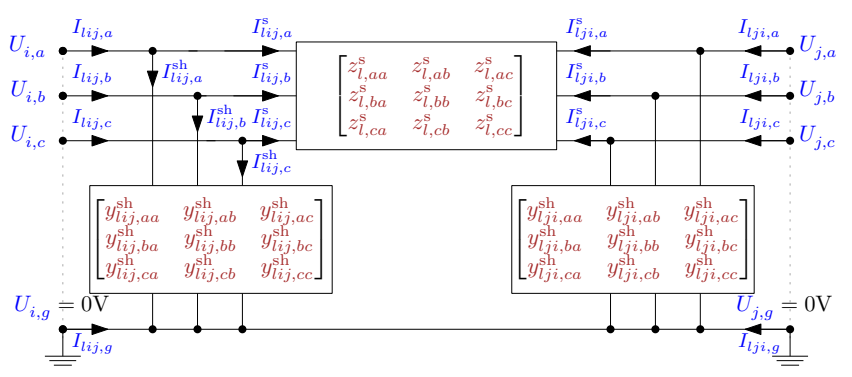

Fig. 1. Unbalanced $3 \times 3 \Pi$-model branch in voltage and current variables.

in the $\mathbf{S}_{l i j}, \mathbf{U}_{i}, \mathbf{I}_{l i j}^{\mathrm{s}}$ variable space. Ohm's law for branch $l$ from node $i$ to $j$, for series impedance $\mathrm{z}_{l}^{\mathrm{s}}$ and from-side shunt admittance $\mathrm{y}_{l i j}^{\text {sh }}$, are formulated in matrix form as

$$
\mathbf{U}_{j}=\mathbf{U}_{i}-\mathrm{z}_{l}^{\mathrm{s}} \mathbf{I}_{l i j}^{\mathrm{s}}, \quad \mathbf{I}_{l i j}^{\mathrm{sh}}=\mathrm{y}_{l i j}^{\mathrm{sh}} \mathbf{U}_{i} .
$$


with

$\mathbf{U}_{i}=\left[\begin{array}{c}U_{i, a} \\ U_{i, b} \\ U_{i, c}\end{array}\right], \mathbf{I}_{l i j}=\left[\begin{array}{c}I_{l i j, a} \\ I_{l i j, b} \\ I_{l i j, c}\end{array}\right], \mathbf{I}_{l i j}^{\mathrm{s}}=\left[\begin{array}{c}I_{l i j, a}^{\mathrm{s}} \\ I_{l i j, b}^{\mathrm{s}} \\ I_{l i j, c}^{\mathrm{s}}\end{array}\right], \mathbf{I}_{l i j}^{\mathrm{sh}}=\left[\begin{array}{c}I_{l i j, a}^{\mathrm{sh}} \\ I_{l i j, b}^{\mathrm{sh}} \\ I_{l i j, c}^{\mathrm{sh}}\end{array}\right]$.

The series power flow $\mathbf{S}_{l i j}^{\mathrm{s}}$ relates to the series current $\mathbf{I}_{l i j}^{\mathrm{s}}$ as,

$$
\mathbf{S}_{l i j}^{\mathrm{s}}=\mathbf{P}_{l i j}^{\mathrm{s}}+j \mathbf{Q}_{l i j}^{\mathrm{s}}=\mathbf{U}_{i}\left(\mathbf{I}_{l i j}^{\mathrm{s}}\right)^{\mathrm{H}} \text {. }
$$

Total and series power flow relate,

$$
\mathbf{S}_{l i j}=\mathbf{U}_{i}\left(\mathbf{U}_{i}\right)^{\mathrm{H}}\left(\mathbf{y}_{l i j}^{\mathrm{sh}}\right)^{\mathrm{H}}+\mathbf{S}_{l i j}^{\mathrm{s}} .
$$

The sum of the power flows through series impedance $\mathrm{z}_{l}^{\mathrm{s}}$ is,

$$
\mathbf{S}_{l i j}^{\mathrm{s}}+\mathbf{S}_{l j i}^{\mathrm{s}}=\mathrm{z}_{l}^{\mathrm{s}} \mathbf{I}_{l i j}^{\mathrm{s}}\left(\mathbf{I}_{l i j}^{\mathrm{s}}\right)^{\mathrm{H}} .
$$

We note that (3), (4) contain only products of voltage and current vectors with themselves.

Voltage magnitudes have minimum and maximum operational limits, which are specific to each conductor and node:

$$
\mathbf{U}_{i}^{\min }=\left[\begin{array}{c}
U_{i, a}^{\min } \\
U_{i, b}^{\min } \\
U_{i, c}^{\min }
\end{array}\right] \leq\left[\begin{array}{l}
\left|U_{i, a}\right| \\
\left|U_{i, b}\right| \\
\left|U_{i, c}\right|
\end{array}\right] \leq\left[\begin{array}{c}
U_{i, a}^{\max } \\
U_{i, b}^{\max } \\
U_{i, c}^{\max }
\end{array}\right]=\mathbf{U}_{i}^{\max } .
$$

This can be written more succinctly as

$$
\mathbf{U}_{i}^{\min } \circ \mathbf{U}_{i}^{\min } \leq \mathbf{U}_{i} \circ\left(\mathbf{U}_{i}\right)^{*} \leq \mathbf{U}_{i}^{\max } \circ \mathbf{U}_{i}^{\max } .
$$

Currents magnitudes should stay below rated values $\mathbf{I}_{l i j}^{\text {rated }}$,

$$
\left[\begin{array}{l}
\left|I_{l i j, a}\right| \\
\left|I_{l i j, b}\right| \\
\left|I_{l i j, c}\right|
\end{array}\right] \leq\left[\begin{array}{c}
I_{l i j, a}^{\text {rated }} \\
I_{l i j, b}^{\text {rated }} \\
I_{l i j, c}^{\text {rated }}
\end{array}\right]=\mathbf{I}_{l i j}^{\text {rated }}
$$

Note that this applied to $\mathbf{I}_{l i j}$ as well. A valid SOC representation of current magnitude limits that does not require explicit current variables $\mathbf{I}_{l i j}$ as,

$$
\left[\begin{array}{c}
\left|S_{l i j, a a}\right|^{2} \\
\left|S_{l i j, b b}\right|^{2} \\
\left|S_{l i j, c c}\right|^{2}
\end{array}\right] \leq\left[\begin{array}{c}
\left(I_{l i j, a}^{\text {rated }}\right)^{2} \\
\left(I_{l i j, b}^{\text {rated }}\right)^{2} \\
\left(I_{l i j, c}^{\text {rated }}\right)^{2}
\end{array}\right] \circ\left[\begin{array}{l}
\left|U_{i, a}\right|^{2} \\
\left|U_{i, b}\right|^{2} \\
\left|U_{i, c}\right|^{2}
\end{array}\right]
$$

which can be developed in matrix notation is,

$$
\operatorname{diag}\left(\mathbf{S}_{l i j}\right) \circ \operatorname{diag}\left(\mathbf{S}_{l i j}\right)^{*} \leq \mathbf{I}_{l i j}^{\mathrm{rated}} \circ \mathbf{I}_{l i j}^{\mathrm{rated}} \circ \mathbf{U}_{i} \circ \mathbf{U}_{i}{ }^{*} .
$$

\section{B. Paper Structure}

First, $\S$ II illustrates lifting and convexification of unbalanced BFM by Gan and Low [6]. Next, §III shows how the derived rank-1-constrained positive semidefinite matrix variables can be reformulated as sets of nonconvex quadratic matrix equations. Therefore, $\S \mathrm{IV}$ provides a numerical comparison of the new formulation with respect to published ones. Finally, $\S \mathrm{V}$ concludes the paper. The Appendix provides in-depth discussion of the underlying derivations, and details on the implementation.

\section{LIFTED UNBALANCED BFM \& CONVEXIFICATION}

This section illustrates the derivation, based on [6], of the lifted BFM and its SDP relaxation.

\section{A. Lifting of Variables}

Products of vectors with their own conjugate transpose (superscript H) generate rank-1 Hermitian matrices. Hermitian matrices have a number of properties which can be exploited. We now discuss the introduction of matrix variables to represent such products.

1) Lifted Node Voltage Variable: A matrix variable for the bus voltage products, $\mathbf{W}_{i}$ is defined as (unique variables are underlined):

$$
\mathbf{W}_{i}=\mathbf{U}_{i} \mathbf{U}_{i}^{\mathrm{H}}=\mathbf{W}_{i}^{\mathrm{re}}+j \mathbf{W}_{i}^{\mathrm{im}} .
$$

This has the following equivalent rectangular scalar representation:

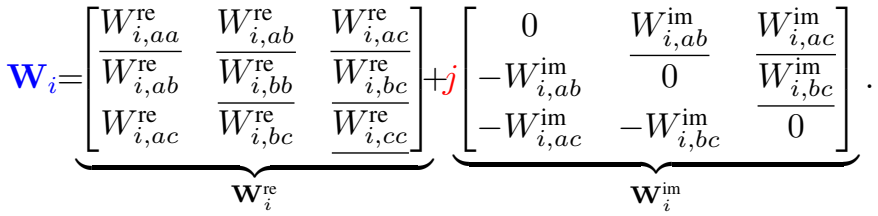

Note that this structure is common to all Hermitian matrices $\mathbb{H}^{n}$. The following statement is an equivalence:

$$
\mathbf{W}_{i}=\mathbf{U}_{i} \mathbf{U}_{i}^{\mathrm{H}} \Longleftrightarrow \mathbf{W}_{i} \succeq 0, \mathbf{W}_{i} \in \mathbb{H}^{n}, \operatorname{rank}\left(\mathbf{W}_{i}\right)=1(11)
$$

Furthermore, this implies that $\operatorname{diag}\left(\mathbf{W}_{i}\right) \geq 0$, which is linear, and a relaxation of the requirement of being positive semidefinite (which is nonlinear, but convex). Bounds (6) can be formulated in terms of $\mathbf{W}_{i}$ using identity $\operatorname{diag}\left(\mathbf{W}_{i}\right)=$ $\mathbf{U}_{i} \circ\left(\mathbf{U}_{i}\right)^{*}$.

2) Series Current Variable: An auxiliary variable for the current products, $\mathbf{L}_{l}^{\mathrm{s}}$ is defined as:

$$
\mathbf{L}_{l}^{\mathrm{s}}=\mathbf{L}_{l}^{\mathrm{s}, \mathrm{re}}+j \mathbf{L}_{l}^{\mathrm{s}, \mathrm{im}}=\mathbf{I}_{l i j}^{\mathrm{s}}\left(\mathbf{I}_{l i j}^{\mathrm{s}}\right)^{\mathrm{H}}=\mathbf{I}_{l j i}^{\mathrm{s}}\left(\mathbf{I}_{l j i}^{\mathrm{s}}\right)^{\mathrm{H}} .
$$

This has the following representation in scalar variables $L$ :

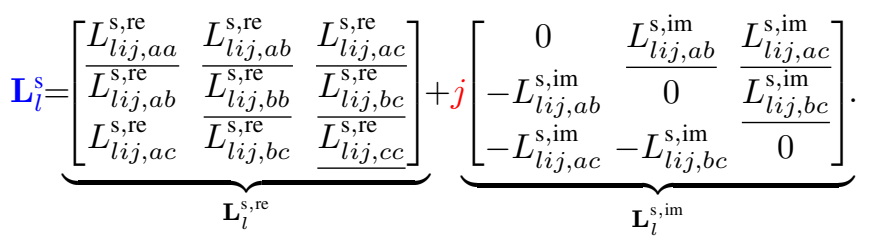

This matrix variable inherits the same PSD Hermitian matrix properties as $\mathbf{W}_{i}$.

\section{B. Lifting of Power Flow Equations}

We now perform substitutions $\mathbf{I}_{l i j}^{\mathrm{s}}\left(\mathbf{I}_{l i j}^{\mathrm{s}}\right)^{\mathrm{H}} \rightarrow \mathbf{L}_{l}^{\mathrm{s}}$ and $\mathbf{U}_{i}\left(\mathbf{U}_{i}\right)^{\mathrm{H}} \rightarrow \mathbf{W}_{i}$ in (4) and (3) and obtain,

$$
\begin{gathered}
\mathbf{S}_{l i j}=\mathbf{W}_{i}\left(\mathbf{y}_{l i j}^{\mathrm{sh}}\right)^{\mathrm{H}}+\mathbf{S}_{l i j}^{\mathrm{s}}, \\
\mathbf{S}_{l i j}^{\mathrm{s}}+\mathbf{S}_{l j i}^{\mathrm{s}}=\mathbf{z}_{l}^{\mathrm{s}} \mathbf{L}_{l}^{\mathrm{s}} .
\end{gathered}
$$

Bounds (9) can be formulated in terms of $\mathbf{W}_{i}$ using identity $\operatorname{diag}\left(\mathbf{W}_{i}\right)=\mathbf{U}_{i} \circ\left(\mathbf{U}_{i}\right)^{*}$. We multiply the LHS and RHS of (1) with their own conjugate transposes and obtain a lifted representation of Ohm's law,

$$
\mathbf{W}_{j}=\mathbf{W}_{i}-\mathbf{S}_{l i j}^{\mathrm{s}}\left(\mathrm{z}_{l}^{\mathrm{s}}\right)^{\mathrm{H}}-\mathbf{z}_{l}^{\mathrm{s}}\left(\mathbf{S}_{l i j}^{\mathrm{s}}\right)^{\mathrm{H}}+\mathrm{z}_{l}^{\mathrm{s}} \mathbf{L}_{l}^{\mathrm{s}}\left(\mathbf{z}_{l}^{\mathrm{s}}\right)^{\mathrm{H}} .
$$


The nonlinear relation (2) between voltage $\mathbf{W}_{i}$, power $\mathbf{S}_{l i j}^{\mathrm{s}}$ and current $\mathbf{L}_{l}^{\mathrm{s}}$ is typically encoded as,

$$
\mathbf{M}_{l i j}=\left[\begin{array}{c}
\mathbf{U}_{i} \\
\mathbf{I}_{l i j}^{\mathrm{s}}
\end{array}\right]\left[\begin{array}{c}
\mathbf{U}_{i} \\
\mathbf{I}_{l i j}^{\mathrm{s}}
\end{array}\right]^{\mathrm{H}}=\left[\begin{array}{cc}
\mathbf{W}_{i} & \mathbf{S}_{l i j}^{\mathrm{s}} \\
\left(\mathbf{S}_{l i j}^{\mathrm{s}}\right)^{\mathrm{H}} & \mathbf{L}_{l}^{\mathrm{s}}
\end{array}\right]
$$

This approach, developed by [7], stacks the current and voltage vectors in a new vector, which is multiplied with its own transpose. The block structure is then identified and mapped to the lifted variable space. From this definition, we obtain properties for all matrices $\mathbf{M}_{l i j}$ :

$$
\mathbf{M}_{l i j} \succeq 0, \operatorname{rank}\left(\mathbf{M}_{l i j}\right)=1
$$

At this step, [7] drops the rank constraints (and therefore the link to the original variable space), and obtains an SDP formulation in the lifted variable space. Gan and Low show how solutions in the original variable space can be recovered from the lifted variable space, if $\operatorname{rank}\left(\mathbf{M}_{l i j}\right)=1$ is observed in the optimum.

\section{Discussion and Paper Scope}

Note that this lifting and reformulation process encodes all the nonconvexities from the original variable space as rank constraints in the lifted one. Solving the relaxed formulation requires a semidefinite programming solver. A variety of solvers is available, including but not limited to, Mosek, SDPA, CSDP, SeDuMi, SDPT3, DSDP. These solvers do not simultaneously support semidefinite constraints/variables, and rank constraints (or any other nonconvex constraints). Therefore, generally, the SDP formulations are benchmarked numerically w.r.t. entirely different formulations, e.g. the AC NLP form in the complex power - polar voltage variable space.

In the balanced case, a lifted but nonconvex formulation exists. For a vector $\mathbf{U}_{i}$ with length one, i.e. $\mathbf{U}_{i}:=\left[U_{i}\right]$ and $\mathbf{I}_{l i j}^{\mathrm{s}}$ with length one, i.e. $\mathbf{I}_{l i j}^{\mathrm{s}}:=\left[I_{l i j}^{\mathrm{s}}\right]$, we know that

$$
\mathbf{M}_{l i j}:=\left[\begin{array}{c}
U_{i} \\
I_{l i j}^{\mathrm{s}}
\end{array}\right]\left[\begin{array}{c}
U_{i} \\
I_{l i j}^{\mathrm{s}}
\end{array}\right]^{\mathrm{H}}=\left[\begin{array}{cc}
W_{i} & P_{l i j}^{\mathrm{s}}+j Q_{l i j}^{\mathrm{s}} \\
P_{l i j}^{\mathrm{s}}-j Q_{l i j}^{\mathrm{s}} & L_{l}^{\mathrm{s}}
\end{array}\right],
$$

which has the equivalent quadratic nonconvex form,

$$
\left(P_{l i j}^{\mathrm{s}}\right)^{2}+\left(Q_{l i j}^{\mathrm{s}}\right)^{2}=W_{i} L_{l}^{\mathrm{s}}
$$

The core contribution of this paper is the development of a matrix generalization of the scalar form (18), to enable an exact solution of the unbalanced power flow equations in the variable space $\mathbf{S}_{l i j}^{\mathrm{s}}, \mathbf{W}_{i}, \mathbf{L}_{l}^{\mathrm{s}}$.

\section{NONCONVEX LIFTED UNBALANCED BFM}

This section defines a quadratic nonconvex form equivalent to the rank-1 constrained SDP variables $\mathbf{M}_{l i j}$.

\section{A. Quadratic Nonconvex Form}

We multiply the definition (2) on the right with its own conjugate transpose, and then apply definitions (10), (12), to come back to the lifted variable space:

$$
\begin{aligned}
\mathbf{S}_{l i j}^{\mathrm{s}}\left(\mathbf{S}_{l i j}^{\mathrm{s}}\right)^{\mathrm{H}} & =\mathbf{U}_{i} \underbrace{\left(\mathbf{I}_{l i j}^{\mathrm{s}}\right)^{\mathrm{H}} \mathbf{I}_{l i j}^{\mathrm{s}}}_{\text {scalar }} \mathbf{U}_{i}{ }^{\mathrm{H}} \\
& =\mathbf{U}_{i}\left(\mathbf{U}_{i}\right)^{\mathrm{H}} \underbrace{\left(\left(\mathbf{I}_{l i j}^{\mathrm{s}}\right)^{\mathrm{H}} \mathbf{I}_{l i j}^{\mathrm{s}}\right)}_{=\operatorname{tr}\left(\mathbf{I}_{l i j}^{\mathrm{s}}\left(\mathbf{I}_{l i j}^{\mathrm{s}}\right)^{\mathrm{H}}\right)} \\
& =\mathbf{W}_{i} \operatorname{tr}\left(\mathbf{L}_{l}^{\mathrm{s}}\right)=\mathbf{W}_{i} \operatorname{tr}\left(\mathbf{L}_{l}^{\mathrm{s}, \mathrm{re}}\right) .
\end{aligned}
$$

Analogous, we obtain an expression by multiplying with the conjugate transpose on the left:

$$
\left(\mathbf{S}_{l i j}^{\mathrm{s}}\right)^{\mathrm{H}} \mathbf{S}_{l i j}^{\mathrm{s}}=\operatorname{tr}\left(\mathbf{W}_{i}\right) \mathbf{L}_{l}^{\mathrm{s}}=\operatorname{tr}\left(\mathbf{W}_{i}^{\mathrm{re}}\right) \mathbf{L}_{l}^{\mathrm{s}} .
$$

The scalar equivalents are illustrated in (22) and (23). Note that we exploit the symmetry, to avoid redundant constraints in the implementation. It is sufficient to post:

- constraints on the diagonal of the real part, i.e. 3 in total;

- constraints on the strict upper triangle of the real part, i.e. 3 in total;

- constraints on the strict upper triangle of the imaginary part, i.e. 3 in total;

therefore 9 constraints for each of (19) and (20). Therefore, the amount of nonconvex quadratic constraints is 18 times the amount of lines.

\section{B. Equivalence in OPF Context}

The Appendix details the mathematical properties of these nonlinear matrix equations, and proves the equivalence between, on the one side (15)-(16), and, on the other side (19)(20), and some requirements on the BFM OPF problem:

- There are no islanded buses or branches with infinite series impedance.

- There exists one bus in the network with a rank-1 $\mathbf{W}_{i}{ }^{1}$. Typically, there is a reference bus, where we fix the phasor $\mathbf{W}_{i}=\mathrm{U}_{i}^{\text {ref }}\left(\mathrm{U}_{i}^{\text {ref }}\right)^{\mathrm{H}}$.

Under these conditions, previously published results for SDP relaxations of OPF with rank-1 exactness conditions, e.g. [6], remain applicable. Therefore, this formulation isn't guaranteed to be exact when solving meshed networks.

The Appendix furthermore derives novel relaxations from the quadratic forms (19)-(20), but concludes that these are dominated by the second-order cone relaxation implied by the $2 \times 2$ principal minors of the complex SDP form.

\section{Solution Recovery}

We use the property $\operatorname{rank}(\mathbf{X})=\operatorname{rank}\left(\mathbf{X X}^{\mathrm{H}}\right)=$ $\operatorname{rank}\left(\mathbf{X}^{\mathrm{H}} \mathbf{X}\right)$. Therefore, together, (19)-(20) conserve rank, i.e.,

$$
\operatorname{rank}\left(\mathbf{S}_{l i j}^{\mathrm{s}}\right)=\operatorname{rank}\left(\mathbf{W}_{i}\right)=\operatorname{rank}\left(\mathbf{L}_{l}^{\mathrm{s}}\right)
$$

although, it isn't guaranteed that the rank will be equal to 1 .

\footnotetext{
${ }^{1}$ This condition is enough to guarantee (30c) holds. Examples include buses with either a fixed voltage, or a balanced phasor with a variable voltage
} magnitude and known angle offsets. 


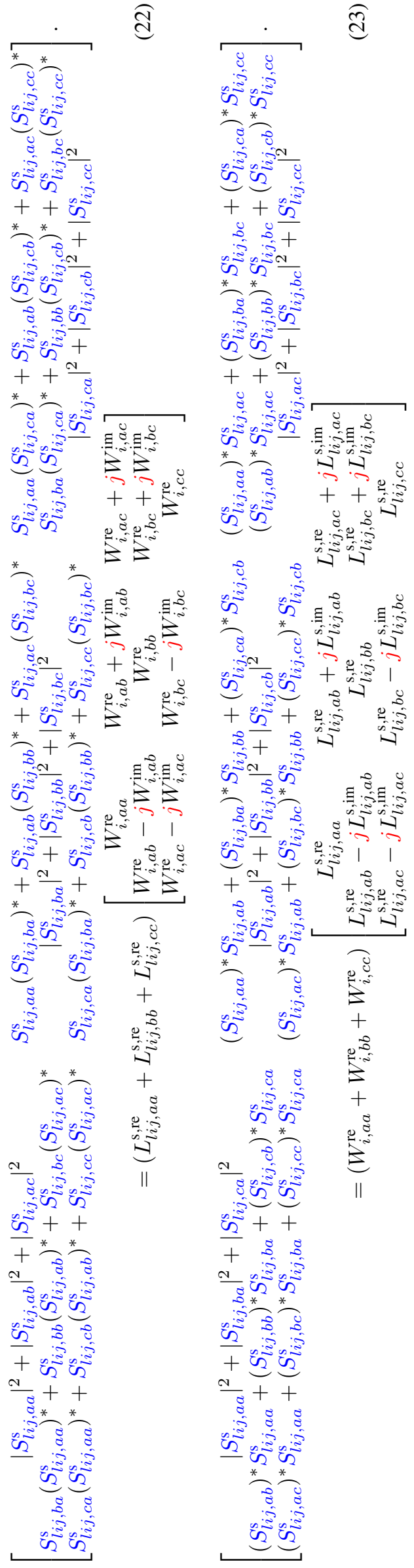

With little loss of generality, we assume there exists a bus in the network with a rank- $1 \mathbf{W}_{i}$, i.e. a reference bus. Therefore, all the branches that connect to that bus will have rank-1 $\mathbf{S}_{l i j}^{\mathrm{s}}$ and $\mathbf{L}_{l}^{\mathrm{s}}$ associated with them. We must now see that the rank-1 property propagates to $\mathbf{S}_{l j i}^{\mathrm{s}}$. In this case, the solution recovery proof is the same as that detailed Lemma 2 in the Appendix of [6] (modification to include the branch shunts as in [8]).

The key argument of that proof is that, if $i$ and $j$ are connected through branch $l$, and if $\operatorname{rank}\left(\mathbf{W}_{i}\right)=1$ then $\operatorname{rank}\left(\mathbf{W}_{j}\right)$ must be 1 as well. The proof details an algorithm, using a known voltage phasor $\mathbf{U}_{i}$, and rank-1 $\mathbf{S}_{l i j}^{\mathrm{s}}$ to derive $\mathbf{I}_{l i j}^{\mathrm{s}}$, which is then used in Ohm's law (1) to derive a $\mathbf{U}_{j}$ that also satisfies the lifted equations. From there on we know another rank-1 bus, i.e. $j$, and the argument repeats until all connected branches and buses have been visited.

\section{NUMERICAL EXPERIMENTS}

\section{A. Implementation}

In real variables, (19)-(20) become,

$$
\begin{aligned}
& \mathbf{P}_{l i j}^{\mathrm{s}}\left(\mathbf{P}_{l i j}^{\mathrm{s}}\right)^{\mathrm{H}}+\mathbf{Q}_{l i j}^{\mathrm{s}}\left(\mathbf{Q}_{l i j}^{\mathrm{s}}\right)^{\mathrm{H}}=\mathbf{W}_{i}^{\mathrm{re}} \operatorname{tr}\left(\mathbf{L}_{l}^{\mathrm{s}, \mathrm{re}}\right), \\
& \mathbf{Q}_{l i j}^{\mathrm{s}}\left(\mathbf{P}_{l i j}^{\mathrm{s}}\right)^{\mathrm{H}}-\mathbf{P}_{l i j}^{\mathrm{s}}\left(\mathbf{Q}_{l i j}^{\mathrm{s}}\right)^{\mathrm{H}}=\mathbf{W}_{i}^{\mathrm{im}} \operatorname{tr}\left(\mathbf{L}_{l}^{\mathrm{s}, \mathrm{re}}\right), \\
& \left(\mathbf{P}_{l i j}^{\mathrm{s}}\right)^{\mathrm{H}} \mathbf{P}_{l i j}^{\mathrm{s}}+\left(\mathbf{Q}_{l i j}^{\mathrm{s}}\right)^{\mathrm{H}} \mathbf{Q}_{l i j}^{\mathrm{s}}=\mathbf{L}_{l}^{\mathrm{s}, \mathrm{re}} \operatorname{tr}\left(\mathbf{W}_{i}^{\mathrm{re}}\right), \\
& \left(\mathbf{P}_{l i j}^{\mathrm{s}}\right)^{\mathrm{H}} \mathbf{Q}_{l i j}^{\mathrm{s}}-\left(\mathbf{Q}_{l i j}^{\mathrm{s}}\right)^{\mathrm{H}} \mathbf{P}_{l i j}^{\mathrm{s}}=\mathbf{L}_{l}^{\mathrm{s}, \mathrm{im}} \operatorname{tr}\left(\mathbf{W}_{i}^{\mathrm{re}}\right),
\end{aligned}
$$

which are nonconvex quadratic equations composed of bilinear and quadratic terms with all coefficients 1/-1. Furthermore, implementation in toolboxes without matrix variable primitives requires scalarization. As the approach can be generalized (see Appendix), the actual implementation is (56a)-(56d). The equations are implemented in the JuMP [9] mathematical programming toolbox in Julia. The numerical results are generated with PowerModelsDistribution ${ }^{2}$, a domain specific toolbox for power distribution network optimization. Table I

\begin{tabular}{|c|c|c|c|c|}
\hline Form & coord & complexity & representation & i solver reference \\
\hline$\overline{\mathrm{ACP}}$ & BIM SU & NLP & trig & Ipopt [11] \\
\hline ACR & BIM SU & NLP & quadr. & Ipopt \\
\hline SDPR & BFM SWL rect. & SDP & conic & Mosek [6] \\
\hline R1SDP & BFM SWL rect. & R-1 SDP & quadr. & Ipopt [6] \\
\hline SOCR & BFM SWL rect. & SOC & conic & Mosek [6], [12] \\
\hline SOCNLP & BFM SWL rect. & SOC & quadr. & Ipopt [6], [12] \\
\hline
\end{tabular}
summarizes the formulations that are compared numerically.

TABLE I

IMPLEMENTED UNBALANCED POWER FLOW FORMULATIONS

\section{B. Solvers and Formulations}

Ipopt 3.12.10 is used as a local solver for the nonconvex quadratic forms [10]; Mosek 9.1.13 is used to solve the SDP forms to global optimality. For Ipopt, we initialize the diagonals of $\mathbf{W}_{i}$ to $1 \mathrm{pu}$ and $\mathbf{L}_{l}^{\mathrm{s}}$ to small but nonzero, positive values (0.01). The rest of the variables are initialized in 0 . Mosek returns results reliably using the default settings. Custom settings are used for Ipopt, i.e. tol=1e-10; acceptable_tol=1e-8, max_iter $=10000$.

\footnotetext{
${ }^{2}$ https://github.com/lanl-ansi/PowerModelsDistribution.jl
} 


\section{Numerical Illustration}

We use two LV test systems, a simple 3-bus radial system, and a modified version of 'LVTestCase'. For both, the source bus is configured with a $230 \mathrm{~V}$ L-N balanced phasor and active/reactive power slack (generator). There is also a generator on an intermediate bus $(1.2 / \mathrm{kW})$, that is more expensive than the (slack) generator on the source bus $(1.0 / \mathrm{kW})$. The objective is to minimize the generator fuel cost (linear). The branches are 4-wire (Kron-reduced) and have small but nonzero shunt admittances. The voltage bounds are 0.9 and $1.1 \mathrm{pu}$ for each bus and each phase.

We normalize the variables using a voltage base of $230 \mathrm{~V}$ and a power base of $1 \mathrm{kVA}$. In general we note that a high number of iterations is required for the R1SDP and SOCNLP forms (up to a few thousand), which is atypical for Ipopt. We note that it was important to set reasonable branch flow bounds for all formulations except for ACP and ACR.

1) Radial 3-Bus System: We use a simple radial 3bus system ${ }^{3}$ (1-source, 2-intermediate, 3-load bus) with significant phase unbalance due to an unbalanced constantpower wye load set to $[9.0,6.0,6.0]^{\mathrm{T}} \mathrm{kW}$ and $[3.0,3.0,3.0]^{\mathrm{T}}$ kvar. The voltage magnitude at the load bus in the optimum is $[0.964,0.982,0.977]^{\mathrm{T}} \mathrm{pu}$ and the angles are $[-0.484,-120.243,120.274]^{\mathrm{T}}$ degrees. Table II gives observed objective values.

TABLE II

OBJECTIVE VALUES FOR 'CASE3_UNBALANCED.DSS'

\begin{tabular}{lllllll}
\hline & ACP & ACR & SDPR & R1SDP & SOCR & SOCRNLP \\
\hline Obj. & 21.4812 & 21.4812 & 21.4812 & 21.4872 & 21.1792 & 21.1787 \\
Gap \% & - & - & - & -0.0280 & 1.4058 & 1.4082 \\
\hline
\end{tabular}

Formulations ACP-ACR are the nonconvex AC polar and rectangular forms, therefore defining upper bounds on the global optimum. They have agreeing objective values; this is used to define the gap. Formulations SDPR, SOCR, SOCNLP are relaxations and provide lower bounds. The SDP relaxation, SDPR, drops the rank-1 constraint but nevertheless obtains an identical objective and decision variables (after solution recovery). Formulation R1SDP is the lifted nonconvex BFM form proposed in this paper and has a small gap; presumably due to numerical challenges. SOCR and SOCRNLP are principal minor SOC relaxations of SDP relaxation SDPR. They should have identical objective values, however conic form SOCR (Mosek) had better convergence than NLP form SOCRNLP (Ipopt). It was important for R1SDP and SOCRNLP to use a small power base, i.e., $1 \mathrm{kVA}$; the other forms weren't that sensitive to the base choice. Nevertheless, even though there is a small gap in R1SDP, decision variables are very similar to those of ACP, ACR, SDPR. It is presumed some of the difference is due to the lower effective accuracy caused by the lifting (taking the square root in post processing magnifies error).

\footnotetext{
${ }^{3}$ https://github.com/lanl-ansi/PowerModelsDistribution.j1/master/test/data/ opendss/case3_unbalanced.dss
}

2) LVTestCase: We take the 'European Low Voltage Test Feeder' a.k.a. 'LVTestCase' from the IEEE Distribution Test Feeders library [13]. To avoid numerical issues, we adapt the case. We merge consecutive branches that don't have loads/generators on the intermediate bus. Furthermore, we remove trailing branches and aggregate the loads/generators to the main feeder. The reduced network size is 118 threephase buses (354 nodes) and 117 branches. We add the second generator on bus 854 (as named in the original data). Table III summarizes the objective values. We note that again, ACP and ACR, agree, and that R1SDP obtains a value that is very close. The SDP relaxation is tight, but we note that no flow bounds are active. Both SOC relaxations have a similar gap, however SOCR takes 31 iterations and SOCRNLP takes 385.

TABLE III

OBJECTIVE VALUES FOR LVTESTCASE

\begin{tabular}{lllllll}
\hline & ACP & ACR & SDPR & R1SDP & SOCR & SOCRNLP \\
\hline Obj. & 49.0272 & 49.0272 & 49.0272 & 49.0209 & 48.3356 & 48.3356 \\
Gap \% & - & - & 0.000 & 0.013 & 1.411 & 1.411 \\
\hline
\end{tabular}

\section{CONCLUSIONS}

A novel formulation of the unbalanced power flow equations is developed, that is exact for radial grids. The formulation is developed in a lifted variable space, but a convex relaxation is not performed. Instead, a set of complex-value nonconvex matrix equations is obtained, reformulated as a set of scalar real-value equations, implemented in JuMP and solved using the NLP solver Ipopt. The numerical results show that this is a workable approach to generate upper bounds on the optimality of unbalanced OPF problems, as the implementation changes w.r.t. SDP relaxation forms are minimal, when using a solver-independent mathematical programming toolbox. The mathematical reformulation technique is generic and can be applied to other formulations, or problems outside of OPF. Numerical challenges were nevertheless observed; analyzing and addressing them is a topic for future work. The development of a library of unbalanced OPF test cases will also enable better comparison of different formulations and solvers.

\section{ACKNOWLEDGEMENT}

The authors thank Thomas Brinsmead, James Foster and Julio Braslavsky for their feedback on the drafts.

\section{APPENDIX}

\section{A. Equivalence of Rank-1-Constrained PSD \& Quadratic Forms}

Two complex vectors are defined

$$
\mathbf{a} \in \mathbb{C}^{n \times 1}, \mathbf{b} \in \mathbb{C}^{m \times 1} .
$$

We construct the block matrix $\mathrm{M}$ as the outer product of a stack of $\mathbf{a}$ and $\mathbf{b}$, with its own conjugate, and partition it,

$$
\mathbf{M}=\left[\begin{array}{l}
\mathbf{a} \\
\mathbf{b}
\end{array}\right]\left[\begin{array}{l}
\mathbf{a} \\
\mathbf{b}
\end{array}\right]^{\mathrm{H}}=\left[\begin{array}{cc}
\mathbf{a a}^{\mathrm{H}} & \mathbf{a b} \mathbf{b}^{\mathrm{H}} \\
\left.(\mathbf{a b})^{\mathrm{H}}\right)^{\mathrm{H}} & \mathbf{b} \mathbf{b}^{\mathrm{H}}
\end{array}\right]=\left[\begin{array}{cc}
\mathbf{A} & \mathbf{C} \\
\mathbf{C}^{\mathrm{H}} & \mathbf{B}
\end{array}\right] .
$$


Note that this definition implies,

$\mathbf{A} \in \mathbb{H}^{n}, \mathbb{S}_{+}^{n}, \mathbf{B} \in \mathbb{H}^{m}, \mathbb{S}_{+}^{m}, \mathbf{C} \in \mathbb{C}^{n \times m}, \mathbf{M} \in \mathbb{H}^{n+m}, \mathbb{S}_{+}^{n+m}(27)$

Using identities (26) we can obtain,

$$
\begin{aligned}
& \mathbf{C C}^{\mathrm{H}}=\mathbf{a} \mathbf{b}^{\mathrm{H}} \mathbf{b a}^{\mathrm{H}}=\mathbf{A} \operatorname{tr}(\mathbf{B}), \\
& \mathbf{C}^{\mathrm{H}} \mathbf{C}=\mathbf{b a}^{\mathrm{H}} \mathbf{a b}^{\mathrm{H}}=\operatorname{tr}(\mathbf{A}) \mathbf{B} .
\end{aligned}
$$

We want to understand the relationship between two forms, $\forall \mathbf{A} \in \mathbb{H}^{n}, \mathbf{B} \in \mathbb{H}^{m}, \mathbf{C} \in \mathbb{C}^{n \times m}$, on the one hand:

$$
\begin{aligned}
& \mathbf{M}=\left[\begin{array}{cc}
\mathbf{A} & \mathbf{C} \\
\mathbf{C}^{\mathrm{H}} & \mathbf{B}
\end{array}\right] \succeq 0, \\
& \operatorname{rank}(\mathbf{M})=1
\end{aligned}
$$

and on the other hand:

$$
\begin{aligned}
& \mathbf{C C}^{\mathrm{H}}=\mathbf{A} \operatorname{tr}(\mathbf{B}), \\
& \mathbf{C}^{\mathrm{H}} \mathbf{C}=\operatorname{tr}(\mathbf{A}) \mathbf{B}, \\
& \operatorname{rank}(\mathbf{A})=1 \text { or } \operatorname{rank}(\mathbf{B})=1 \text { or } \operatorname{rank}(\mathbf{C})=1 . \\
& \operatorname{diag}(\mathbf{A}) \geq 0, \operatorname{diag}(\mathbf{B}) \geq 0 .
\end{aligned}
$$

It will be shown that both forms are equivalent. Note that in either case, $\mathbf{C}$ is not unique, as both forms allow for an arbitrary scalar rotation of that matrix. Throughout the proof, we will assume that some unique $\mathbf{a} \in \mathbb{C}^{n \times 1}, \mathbf{b} \in \mathbb{C}^{m \times 1}$ exist.

1) Implication: The rank-1-ness, Hermitian structure, and PSD-ness of $M$ guarantee that it can be factored into a vector times its own conjugate transpose. Given the block structure of $\mathbf{M}$, such vector is composed of component vectors $\overline{\mathbf{a}}, \overline{\mathbf{b}}$ :

$$
\left[\begin{array}{cc}
\mathbf{A} & \mathbf{C} \\
\mathbf{C}^{\mathrm{H}} & \mathbf{B}
\end{array}\right]=\left[\begin{array}{l}
\overline{\mathbf{a}} \\
\overline{\mathbf{b}}
\end{array}\right]\left[\begin{array}{l}
\overline{\mathbf{a}} \\
\overline{\mathbf{b}}
\end{array}\right]^{\mathrm{H}} .
$$

From here on, one repeats the steps in (28a) and (28b) for $\overline{\mathbf{a}}$ and $\overline{\mathrm{b}}$, which proves the implication.

2) Converse implication: Using $\operatorname{rank}(\mathbf{X})=$ $\operatorname{rank}\left(\mathbf{X X}^{\mathrm{H}}\right)=\operatorname{rank}\left(\mathbf{X}^{\mathrm{H}} \mathbf{X}\right)$ and $\operatorname{rank}(a \mathbf{X})=\operatorname{rank}(\mathbf{X}), a \neq$ 0 , and applying it to the LHS and RHS of (30a) (30b), in combination with (30c), we find,

$$
\operatorname{rank}(\mathbf{A})=\operatorname{rank}(\mathbf{B})=\operatorname{rank}(\mathbf{C})=1 .
$$

Both $\mathbf{A}$ and $\mathbf{B}$ are rank-1, Hermitian and have nonnegative diagonals, which guarantees the existence of vectors $\mathbf{a}^{\prime}$ and $\mathbf{b}^{\prime}$ which satisfy

$$
\exists \mathbf{a}^{\prime}: \mathbf{A}=\mathbf{a}^{\prime}\left(\mathbf{a}^{\prime}\right)^{\mathrm{H}}, \exists \mathbf{b}^{\prime}: \mathbf{B}=\mathbf{b}^{\prime}\left(\mathbf{b}^{\prime}\right)^{\mathrm{H}} .
$$

These are part of families of vectors, unique up to a unit rotation. If solutions $\mathbf{a}$ and $\mathbf{b}$ exist, they must therefore be rotations of $\mathbf{a}^{\prime}$ and $\mathbf{b}^{\prime}$ which we parameterize by $\theta_{a}, \theta_{b}$,

$$
\exists \theta_{a} \in \mathbb{R}: \mathbf{a}^{\prime}=e^{j \theta_{a}} \mathbf{a}, \exists \theta_{b} \in \mathbb{R}: \mathbf{b}^{\prime}=e^{j \theta_{b}} \mathbf{b} .
$$

We can work out the RHS of (30a) (30b), and see that it is invariant to unit rotation $e^{j \theta_{c}}$,

$$
\begin{aligned}
\operatorname{Atr}(\mathbf{B}) & =\mathbf{a}^{\prime}\left(\mathbf{a}^{\prime}\right)^{\mathrm{H}}\left(\mathbf{b}^{\prime}\right)^{\mathrm{H}} \mathbf{b}^{\prime}=\left(\mathbf{a}^{\prime}\left(\mathbf{b}^{\prime}\right)^{\mathrm{H}}\right)\left(\mathbf{a}^{\prime}\left(\mathbf{b}^{\prime}\right)^{\mathrm{H}}\right)^{\mathrm{H}}, \\
& =\left(e^{j \theta_{c}} \mathbf{a}^{\prime}\left(\mathbf{b}^{\prime}\right)^{\mathrm{H}}\right)\left(e^{j \theta_{c}} \mathbf{a}^{\prime}\left(\mathbf{b}^{\prime}\right)^{\mathrm{H}}\right)^{\mathrm{H}}, \\
\operatorname{tr}(\mathbf{A}) \mathbf{B} & =\left(\mathbf{a}^{\prime}\right)^{\mathrm{H}} \mathbf{a}^{\prime} \mathbf{b}^{\prime}\left(\mathbf{b}^{\prime}\right)^{\mathrm{H}}=\left(\mathbf{a}^{\prime}\left(\mathbf{b}^{\prime}\right)^{\mathrm{H}}\right)^{\mathrm{H}}\left(\mathbf{a}^{\prime}\left(\mathbf{b}^{\prime}\right)^{\mathrm{H}}\right), \\
& =\left(e^{j \theta_{c}} \mathbf{a}^{\prime}\left(\mathbf{b}^{\prime}\right)^{\mathrm{H}}\right)^{\mathrm{H}}\left(e^{j \theta_{c}} \mathbf{a}^{\prime}\left(\mathbf{b}^{\prime}\right)^{\mathrm{H}}\right) .
\end{aligned}
$$

and now define a new matrix $\mathbf{C}^{\prime}$.

$$
\mathbf{C}^{\prime}=e^{j \theta_{c}} \mathbf{a}^{\prime}\left(\mathbf{b}^{\prime}\right)^{\mathrm{H}}=e^{j \theta_{c}} e^{j\left(\theta_{a}-\theta_{b}\right)} \mathbf{a b}^{\mathrm{H}}=e^{j \theta_{c}} e^{j\left(\theta_{a}-\theta_{b}\right)} \mathbf{C} .
$$

We didn't set our to recover $\mathbf{C}$ uniquely, so by setting $\theta_{c}$ to 0 going forward, we accept that we will instead find a unit rotated version of $\mathbf{C}$. We define diagonal matrix $\mathbf{R}$ as

$$
\mathbf{R}=\operatorname{diagm}\left(\left[\begin{array}{l}
e^{j \theta_{a}} \mathbf{1}_{n} \\
e^{j \theta_{b}} \mathbf{1}_{m}
\end{array}\right]\right),
$$

where diagm returns a matrix with the given vector on the diagonal. We can now construct $\mathbf{M}^{\prime}$ analogous to (31)

$$
\begin{gathered}
\mathbf{M}^{\prime}=\left[\begin{array}{c}
e^{j \theta_{a}} \mathbf{a} \\
e^{j \theta_{b}} \mathbf{b}
\end{array}\right]\left[\begin{array}{c}
e^{j \theta_{a}} \mathbf{a} \\
e^{j \theta_{b}} \mathbf{b}
\end{array}\right]^{\mathrm{H}} \\
=\left[\begin{array}{cc}
\mathbf{A} & e^{j\left(\theta_{a}-\theta_{b}\right)} \mathbf{C} \\
\left(e^{j\left(\theta_{a}-\theta_{b}\right)} \mathbf{C}\right)^{\mathrm{H}} & \mathbf{B}
\end{array}\right] \\
=\mathbf{R} \mathbf{M} \mathbf{R}^{\mathrm{H}}
\end{gathered}
$$

This implies $\mathbf{M}^{\prime} \succeq 0 \Longleftrightarrow \mathbf{M} \succeq 0$, as $\mathbf{R}$ is full rank and unitary $\left(\mathbf{R} \mathbf{R}^{\mathrm{H}}=\mathbf{R}^{\mathrm{H}} \mathbf{R}=\mathbf{I}\right)$, and we also obtain,

$$
\mathbf{M}=\mathbf{R}^{\mathrm{H}} \mathbf{M}^{\prime} \mathbf{R},
$$

which guarantees $\operatorname{rank}(\mathbf{M})=\operatorname{rank}\left(\mathbf{M}^{\prime}\right)=1$.

3) Solution Recovery: Recovery of the original $\mathbf{a}$ and $\mathbf{b}$ is important. In general $\mathbf{a} \neq \mathbf{a}^{\prime}$ and $\mathbf{b} \neq \mathbf{b}^{\prime}$, however, they do belong to families of solutions, characterized by unit rotation. There are three degrees of freedom to resolve, i.e. $\theta_{a}, \theta_{b}, \theta_{c}$ :

$$
\mathbf{a}=e^{-j \theta_{a}} \mathbf{a}^{\prime}, \mathbf{b}=e^{-j \theta_{b}} \mathbf{b}^{\prime}, \mathbf{C}=e^{-j \theta_{c}} e^{-j\left(\theta_{a}-\theta_{b}\right)} \mathbf{C}^{\prime},
$$

which then define a unique solution. Note that recovery of specific $\mathbf{a}$ and $\mathbf{b}$ is done after solving (i.e. the variables were substituted out), however $\mathbf{C}$ was defined in the lifted variable space. Therefore, it can be subject to other equalities, which may already imply uniqueness of $\mathbf{C}^{4}$.

Note that (28a) and (28b) are fourth degree polynomials in the elements of $\mathbf{a}$ and $\mathbf{b}$, and therefore loss of accuracy is expected in solution recovery subject to finite precision arithmetic.

4) Use as Reformulation Technique: The ultimate use of these techniques is the lifting of the vector products:

- outer product $\mathbf{C}=\mathbf{a} b^{\mathrm{H}}$;

- element-wise product $\mathbf{c}=\mathbf{a} \circ \mathbf{b}^{*}$, alternatively $\mathbf{c}=$ $\operatorname{diag}\left(\mathbf{a} \mathbf{b}^{\mathrm{H}}\right)$;

to a variable space in which the nonlinearity causes fewer challenges. This construction therefore generalizes the classic result in SDP reformulation (through $\mathbf{a}:=1$ ), which enables the reformulation of the product of vectors with their own conjugate:

$$
\begin{gathered}
\mathbf{M}=\left[\begin{array}{l}
1 \\
\mathbf{b}
\end{array}\right]\left[\begin{array}{l}
1 \\
\mathbf{b}
\end{array}\right]^{\mathrm{H}}=\left[\begin{array}{cc}
1 & \mathbf{b}^{\mathrm{H}} \\
\mathbf{b} & \mathbf{B}
\end{array}\right], \\
\mathbf{M} \succeq 0, \operatorname{rank}(\mathbf{M})=1 \Longleftrightarrow \mathbf{B}=\mathbf{b b}^{\mathrm{H}} .
\end{gathered}
$$

${ }^{4}$ for instance, in OPF, $\mathbf{C}$ represents $\mathbf{S}_{l i j}^{\mathrm{s}}$, which appears in the power balance equalities at the buses. Eventually, some of those equalities involve loads and/or generators with active and reactive power set points, thereby resolving the unit rotation. 
Note that rank-1 constraints can be translated to sets of quadratic nonconvex polynomials, per Proposition 3.1 in [14]. This states that if an Hermitian matrix $\mathbf{X}$ is PSD and rank-1, if and only if all the $2 \times 2$ minors of $\mathbf{X}$ are zero and the diagonal elements of $\mathbf{X}$ are nonnegative.

Note that a necessary and sufficient condition for an $n \times n$ Hermitian matrix to be PSD is that all its principal minors are nonnegative. Per [15], the total number of principal minors is $2^{n}-1$. They all have maximum polynomial degree of $n$. Conversely, (30a), (30b), show that a nonconvex quadratic polynomial representation of a rank-1 PSD requirement exists, independent of the size of $\mathrm{M}$, and without needing to introduce new variables.

5) Scalar Parameterization: The set of all coordinates in a matrix $\mathbf{X} \in \mathbb{C}^{n \times m}$ is defined

$$
\mathcal{A}(\mathbf{X})=\{1, \ldots, n\} \times\{1, \ldots, m\} .
$$

We define sets $\mathcal{U}(\mathbf{X})$ and $\mathcal{D}(\mathbf{X})$, that respectively contain the coordinates of the upper triangle (strict) and diagonal elements:

$$
\begin{aligned}
& \mathcal{U}(\mathbf{X})=\{(a, b) \mid(a, b) \in \mathcal{A}(\mathbf{X}), a<b\}, \\
& \mathcal{D}(\mathbf{X})=\{(a, a) \mid(a, b) \in \mathcal{A}(\mathbf{X}), a=b\} .
\end{aligned}
$$

The union set is

$$
\mathcal{T}(\mathbf{X})=\mathcal{U}(\mathbf{X}) \cup \mathcal{D}(\mathbf{X})
$$

Note that in the case where $\mathbf{X}=\mathbf{X}^{\mathrm{re}}+j \mathbf{X}^{\mathrm{im}}$ is Herimitian, $\mathcal{T}\left(\mathbf{X}^{\mathrm{re}}\right)$ extracts the unique scalar variables for the real part and $\mathcal{U}\left(\mathbf{X}^{\mathrm{im}}\right)$ for the imaginary part, thereby avoiding redundant constraints. The element at the index $i j$ in the matrix $\mathbf{X}$ is denoted $[\mathbf{X}]_{i j}$.

Using this notation, we can finally define the complex-value parameterized scalar forms,

$$
\begin{aligned}
& \forall(a, b) \in \mathcal{T}(\mathbf{A}): \sum_{j=1: m}[\mathbf{C}]_{a j}\left([\mathbf{C}]_{b j}\right)^{*}=[\mathbf{A}]_{a b} \sum_{j=1: m}[\mathbf{B}]_{j j} \\
& \forall(a, b) \in \mathcal{T}(\mathbf{B}): \sum_{i=1: n}\left([\mathbf{C}]_{i a}\right)^{*}[\mathbf{C}]_{i b}=[\mathbf{B}]_{a b} \sum_{i=1: n}[\mathbf{A}]_{i i} .
\end{aligned}
$$

Note that the equations are symmetrical when you swap $\mathbf{A}$ and $\mathbf{B}$ and replace $\mathbf{C}$ by its own conjugate transpose.

6) Discussion: Equations (29a) are semi-definite programming representable, whereas (30a), (30b) are quadratic nonconvex polynomials. Note that $\mathbf{C C}^{\mathrm{H}} \in \mathbb{H}^{n}$ and $\mathbf{C}^{\mathrm{H}} \mathbf{C} \in \mathbb{H}^{m}$, which means there are a lot of redundant equations. The rank constraints in the polynomial form are much smaller, as the dimension of the matrix to which it applies is $\min (n, m)$ instead of $n+m$. Without rank constraints, in the PSD form (29a), the ranks of $\mathbf{A}, \mathbf{B}$ and $\mathbf{C}$ may differ. This is not the case for the polynomial form, as the ranks are identical, even when not rank-1.

7) SOC Relaxation (Complex): In both representations, one can drop the rank constraints. In the polynomial form, one can see that the diagonals of (30a), (30b) are 1) sums of magnitudes squared on the LHS, and 2) bilinear in nonnegative variables on the RHS, as the diagonal elements of $\mathbf{A}, \mathbf{B}$ are nonnegative and real. A SOC relaxation can therefore be developed without (additional) lift-and-project:

$$
\begin{aligned}
& \operatorname{diag}\left(\mathbf{C C}^{\mathrm{H}}\right) \leq \operatorname{diag}(\mathbf{A}) \operatorname{tr}(\mathbf{B}), \\
& \operatorname{diag}\left(\mathbf{C}^{\mathrm{H}} \mathbf{C}\right) \leq \operatorname{tr}(\mathbf{A}) \operatorname{diag}(\mathbf{B}),
\end{aligned}
$$

The obtained SOC inequalities have scalar forms,

$$
\begin{aligned}
& \forall i: \sum_{j=1: m}\left|[\mathbf{C}]_{i j}\right|^{2} \leq[\mathbf{A}]_{i i} \sum_{j=1: m}[\mathbf{B}]_{j j}, \\
& \forall j: \sum_{i=1: n}\left|[\mathbf{C}]_{i j}\right|^{2} \leq[\mathbf{B}]_{j j} \sum_{i=1: n}[\mathbf{A}]_{i i} .
\end{aligned}
$$

Independently, the SDP form (29a) can be further relaxed to a set of lower-dimensional SDP forms based on the PSD-ness of the principal minors [12]. For a relaxation based on $2 \times 2$ principal minors, the constraints are SOCs. We focus on the SOCs which link $\mathbf{A}, \mathbf{B}$ and $\mathbf{C}$ :

$$
\begin{aligned}
\forall(i, j) & \in \mathcal{A}(\mathbf{C}):\left[\begin{array}{cc}
{[\mathbf{A}]_{i i}} & {[\mathbf{C}]_{i j}} \\
{\left[\mathbf{C}^{\mathrm{H}}\right]_{j i}} & {[\mathbf{B}]_{j j}}
\end{array}\right] \succeq 0 \\
& \Longleftrightarrow\left|[\mathbf{C}]_{i j}\right|^{2} \leq[\mathbf{A}]_{i i}[\mathbf{B}]_{j j} .
\end{aligned}
$$

It is easy to see that (53) implies (52a), (52b) but not the other way around.

8) Real-Value Forms: We define the following matrices to represent the real and imaginary parts of the complex matrices.

$$
\mathbf{A}=\mathbf{A}^{\mathrm{re}}+j \mathbf{A}^{\mathrm{im}}, \mathbf{B}=\mathbf{B}^{\mathrm{re}}+j \mathbf{B}^{\mathrm{im}}, \mathbf{C}=\mathbf{C}^{\mathrm{re}}+j \mathbf{C}^{\mathrm{im}} .
$$

A $n \times n$ Hermitian matrix has $n$ real diagonal elements, $\frac{n^{2}-n}{2}$ upper triangle real elements and $\frac{n^{2}-n}{2}$ upper triangle imaginary elements, so in total, $n^{2}$ real scalar variables.

The nonlinear matrix equations can be expanded as

$$
\begin{aligned}
& \mathbf{C}^{\mathrm{re}}\left(\mathbf{C}^{\mathrm{re}}\right)^{\mathrm{T}}+\mathbf{C}^{\mathrm{im}}\left(\mathbf{C}^{\mathrm{im}}\right)^{\mathrm{T}}=\mathbf{A}^{\mathrm{re}} \operatorname{tr}\left(\mathbf{B}^{\mathrm{re}}\right), \\
& \mathbf{C}^{\mathrm{im}}\left(\mathbf{C}^{\mathrm{re}}\right)^{\mathrm{T}}-\mathbf{C}^{\mathrm{re}}\left(\mathbf{C}^{\mathrm{im}}\right)^{\mathrm{T}}=\mathbf{A}^{\mathrm{im}} \operatorname{tr}\left(\mathbf{B}^{\mathrm{re}}\right), \\
& \left(\mathbf{C}^{\mathrm{re}}\right)^{\mathrm{T}} \mathbf{C}^{\mathrm{re}}+\left(\mathbf{C}^{\mathrm{im}}\right)^{\mathrm{T}} \mathbf{C}^{\mathrm{im}}=\operatorname{tr}\left(\mathbf{A}^{\mathrm{re}}\right) \mathbf{B}^{\mathrm{re}}, \\
& \left(\mathbf{C}^{\mathrm{im}}\right)^{\mathrm{T}} \mathbf{C}^{\mathrm{re}}-\left(\mathbf{C}^{\mathrm{re}}\right)^{\mathrm{T}} \mathbf{C}^{\mathrm{im}}=\operatorname{tr}\left(\mathbf{A}^{\mathrm{re}}\right) \mathbf{B}^{\mathrm{im}},
\end{aligned}
$$

Scalarized, the equations have the following parameterization

$$
\begin{aligned}
& \forall(a, b) \in \mathcal{T}\left(\mathbf{A}^{\mathrm{re}}\right): \sum_{j=1: m}\left[\mathbf{C}^{\mathrm{re}}\right]_{a j}\left[\mathbf{C}^{\mathrm{re}}\right]_{b j}+\left[\mathbf{C}^{\mathrm{im}}\right]_{a j}\left[\mathbf{C}^{\mathrm{im}}\right]_{b j} \\
& =\left[\mathbf{A}^{\mathrm{re}}\right]_{a b} \sum_{j=1: m}\left[\mathbf{B}^{\mathrm{re}}\right]_{j j}, \\
& \forall(a, b) \in \mathcal{U}\left(\mathbf{A}^{\mathrm{im}}\right): \sum_{j=1: m}\left[\mathbf{C}^{\mathrm{im}}\right]_{a j}\left[\mathbf{C}^{\mathrm{re}}\right]_{b j}-\left[\mathbf{C}^{\mathrm{re}}\right]_{a j}\left[\mathbf{C}^{\mathrm{im}}\right]_{b j} \\
& =\left[\mathbf{A}^{\mathrm{im}}\right]_{a b} \sum_{j=1: m}\left[\mathbf{B}^{\mathrm{re}}\right]_{j j}, \\
& \forall(a, b) \in \mathcal{T}\left(\mathbf{B}^{\mathrm{re}}\right): \sum_{i=1: n}\left[\mathbf{C}^{\mathrm{re}}\right]_{i a}\left[\mathbf{C}^{\mathrm{re}}\right]_{i b}+\left[\mathbf{C}^{\mathrm{im}}\right]_{i a}\left[\mathbf{C}^{\mathrm{im}}\right]_{i b} \\
& =\left[\mathbf{B}^{\mathrm{re}}\right]_{a b} \sum_{i=1: n}\left[\mathbf{A}^{\mathrm{re}}\right]_{i i}, \\
& \forall(a, b) \in \mathcal{U}\left(\mathbf{B}^{\mathrm{im}}\right): \sum_{i=1: n}\left[\mathbf{C}^{\mathrm{im}}\right]_{i a}\left[\mathbf{C}^{\mathrm{re}}\right]_{i b}-\left[\mathbf{C}^{\mathrm{re}}\right]_{i a}\left[\mathbf{C}^{\mathrm{im}}\right]_{i b} \\
& =\left[\mathbf{B}^{\mathrm{im}}\right]_{a b} \sum_{i=1: n}\left[\mathbf{A}^{\mathrm{re}}\right]_{i i},
\end{aligned}
$$


Together, these define $n^{2}+m^{2}$ unique real-value scalar equations.

9) SOC Relaxation (Real): In the reals, (53) becomes

$$
\forall(i, j) \in \mathcal{A}\left(\mathbf{C}^{\mathrm{re}}\right):\left(\left[\mathbf{C}^{\mathrm{re}}\right]_{i j}\right)^{2}+\left(\left[\mathbf{C}^{\mathrm{im}}\right]_{i j}\right)^{2} \leq\left[\mathbf{A}^{\mathrm{re}}\right]_{i i}\left[\mathbf{B}^{\mathrm{re}}\right]_{j j}
$$

Eqs. (52a) (52b) in real values become

$$
\begin{aligned}
& \forall i: \sum_{j=1: m}\left(\left[\mathbf{C}^{\mathrm{re}}\right]_{i j}\right)^{2}+\left(\left[\mathbf{C}^{\mathrm{im}}\right]_{i j}\right)^{2} \leq\left[\mathbf{A}^{\mathrm{re}}\right]_{i i} \sum_{j=1: m}\left[\mathbf{B}^{\mathrm{re}}\right]_{j j},(58 \mathrm{a}) \\
& \forall j: \sum_{i=1: n}\left(\left[\mathbf{C}^{\mathrm{re}}\right]_{i j}\right)^{2}+\left(\left[\mathbf{C}^{\mathrm{im}}\right]_{i j}\right)^{2} \leq\left[\mathbf{B}^{\mathrm{re}}\right]_{j j} \sum_{i=1: n}\left[\mathbf{A}^{\mathrm{re}}\right]_{i i} \cdot(58 \mathrm{~b})
\end{aligned}
$$

A real-value form [16] (using the isomorphism between $\mathbb{C}$ and $\mathbb{R}^{2 \times 2}$ ) of (29a) is

$$
\begin{aligned}
& \mathbf{M}^{2, \mathrm{re}}= {\left[\begin{array}{cccc}
\mathbf{A}^{\mathrm{re}} & \mathbf{C}^{\mathrm{re}} & \mathbf{A}^{\mathrm{im}} & \mathbf{C}^{\mathrm{im}} \\
\left(\mathbf{C}^{\mathrm{re}}\right)^{\mathrm{T}} & \mathbf{B}^{\mathrm{re}} & -\left(\mathbf{C}^{\mathrm{im}}\right)^{\mathrm{T}} & \mathbf{B}^{\mathrm{im}} \\
-\mathbf{A}^{\mathrm{im}} & -\mathbf{C}^{\mathrm{im}} & \mathbf{A}^{\mathrm{re}} & \mathbf{C}^{\mathrm{re}} \\
\left(\mathbf{C}^{\mathrm{im}}\right)^{\mathrm{T}} & -\mathbf{B}^{\mathrm{im}} & \left(\mathbf{C}^{\mathrm{re}}\right)^{\mathrm{T}} & \mathbf{B}^{\mathrm{re}}
\end{array}\right] \succeq 0, } \\
& \mathbf{M}^{2, \mathrm{re}} \in \mathbb{S}_{+}^{2(m+n)}, \mathbb{R}^{2(m+n) \times 2(m+n)}
\end{aligned}
$$

The SOCs derived from this form are,

$$
\begin{aligned}
\forall(i, j) & \in \mathcal{A}\left(\mathbf{C}^{\mathrm{re}}\right):\left[\begin{array}{cc}
{\left[\mathbf{A}^{\mathrm{re}}\right]_{i i}} & {\left[\mathbf{C}^{\mathrm{re}}\right]_{i j}} \\
{\left[\left(\mathbf{C}^{\mathrm{re}}\right)^{\mathrm{H}}\right]_{j i}} & {\left[\mathbf{B}^{\mathrm{re}}\right]_{j j}}
\end{array}\right] \succeq 0 \\
& \Longleftrightarrow\left(\left[\mathbf{C}^{\mathrm{re}}\right]_{i j}\right)^{2} \leq\left[\mathbf{A}^{\mathrm{re}}\right]_{i i}\left[\mathbf{B}^{\mathrm{re}}\right]_{j j}, \\
\forall(i, j) & \in \mathcal{A}\left(\mathbf{C}^{\mathrm{re}}\right):\left[\begin{array}{cc}
{\left[\mathbf{A}^{\mathrm{re}}\right]_{i i}} & {\left[\mathbf{C}^{\mathrm{im}}\right]_{i j}} \\
{\left[\left(\mathbf{C}^{\mathrm{im}}\right)^{\mathrm{H}}\right]_{j i}} & {\left[\mathbf{B}^{\mathrm{re}}\right]_{j j}}
\end{array}\right] \succeq 0 \\
& \Longleftrightarrow\left(\left[\mathbf{C}^{\mathrm{im}}\right]_{i j}\right)^{2} \leq\left[\mathbf{A}^{\mathrm{re}}\right]_{i i}\left[\mathbf{B}^{\mathrm{re}}\right]_{j j} .
\end{aligned}
$$

We can sum (60a), (60b) over $i$ or $j$ to obtain:

$$
\begin{aligned}
& \forall i: \sum_{j=1: m}\left(\left[\mathbf{C}^{\mathrm{re}}\right]_{i j}\right)^{2}+\left(\left[\mathbf{C}^{\mathrm{im}}\right]_{i j}\right)^{2} \leq 2\left(\left[\mathbf{A}^{\mathrm{re}}\right]_{i i} \sum_{j=1: m}\left[\mathbf{B}^{\mathrm{re}}\right]_{j j}\right),(61 \mathrm{a}) \\
& \forall j: \sum_{i=1: n}\left(\left[\mathbf{C}^{\mathrm{re}}\right]_{i j}\right)^{2}+\left(\left[\mathbf{C}^{\mathrm{im}}\right]_{i j}\right)^{2} \leq 2\left(\left[\mathbf{B}^{\mathrm{re}}\right]_{j j} \sum_{i=1: n}\left[\mathbf{A}^{\mathrm{re}}\right]_{i i}\right),(61 \mathrm{~b})
\end{aligned}
$$

The tightest real-value SOC form is (57), which implies (58a), (58b) as well as (60a), (60b). Forms (58a), (58b) and (60a), (60b) both imply (61a), (61b), however, they are otherwise incomparable.

10) Bounds: We assume the following bounds on the elements of $\mathbf{a}$ and $\mathbf{b}$.

$$
\begin{aligned}
& 0 \leq \mathrm{a}^{\min } \leq|\mathbf{a}| \leq \mathrm{a}^{\max } \\
& 0 \leq \mathrm{b}^{\min } \leq|\mathbf{b}| \leq \mathrm{b}^{\max }
\end{aligned}
$$

and on $\mathbf{c}=\mathbf{c}^{\mathrm{re}}+j \mathbf{c}^{\mathrm{im}}$.

$$
\begin{gathered}
\mathrm{c}^{\mathrm{re}, \min } \leq \mathbf{c}^{\mathrm{re}} \leq \mathrm{c}^{\mathrm{re}, \max }, \\
\mathrm{c}^{\mathrm{im}, \min } \leq \mathbf{c}^{\mathrm{im}} \leq \mathrm{c}^{\mathrm{im}, \max }, \\
0 \leq|\mathbf{c}| \leq \mathbf{c}^{\max }
\end{gathered}
$$

Then the following constraints are valid and convex inequalities on the matrix variables $\mathbf{A}, \mathbf{B}$ and $\mathbf{C}$,

$$
\begin{gathered}
-\mathbf{a}^{\max }\left(\mathbf{a}^{\max }\right)^{\mathrm{T}} \leq \mathbf{A}^{\mathrm{re}}, \mathbf{A}^{\mathrm{im}} \leq \mathbf{a}^{\max }\left(\mathbf{a}^{\max }\right)^{\mathrm{T}} \\
\mathbf{a}^{\min } \circ \mathbf{a}^{\min } \leq \operatorname{diag}\left(\mathbf{A}^{\mathrm{re}}\right) \leq \mathbf{a}^{\max } \circ \mathbf{a}^{\max }, \\
-\mathbf{b}^{\max }\left(\mathbf{b}^{\max }\right)^{\mathrm{T}} \leq \mathbf{B}^{\mathrm{re}}, \mathbf{B}^{\mathrm{im}} \leq \mathbf{b}^{\max }\left(\mathbf{b}^{\max }\right)^{\mathrm{T}} \\
\mathbf{b}^{\min } \circ \mathbf{b}^{\min } \leq \operatorname{diag}\left(\mathbf{B}^{\mathrm{re}}\right) \leq \mathbf{b}^{\max } \circ \mathbf{b}^{\max }, \\
-\mathbf{a}^{\max }\left(\mathbf{b}^{\max }\right)^{\mathrm{T}} \leq \mathbf{C}^{\mathrm{re}}, \mathbf{C}^{\mathrm{im}} \leq \mathbf{a}^{\max }\left(\mathbf{b}^{\max }\right)^{\mathrm{T}}, \\
\mathbf{c}^{\mathrm{re}, \min } \leq \operatorname{diag}\left(\mathbf{C}^{\mathrm{re}}\right) \leq \mathbf{c}^{\mathrm{re}, \max }, \\
\mathbf{c}^{\mathrm{im}, \min } \leq \operatorname{diag}\left(\mathbf{C}^{\text {im }}\right) \leq \mathbf{c}^{\mathrm{im}, \max }, \\
\operatorname{diag}\left(\mathbf{C}^{\mathrm{re}}\right) \circ \operatorname{diag}\left(\mathbf{C}^{\mathrm{re}}\right)+\operatorname{diag}\left(\mathbf{C}^{\mathrm{re}}\right) \circ \operatorname{diag}\left(\mathbf{C}^{\mathrm{re}}\right) \\
\leq \mathbf{c}^{\max } \circ \mathbf{c}^{\max } .
\end{gathered}
$$

\section{REFERENCES}

[1] D. K. Molzahn and I. A. Hiskens, "Mixed SDP / SOCP moment relaxations of the optimal power flow problem," in IEEE Powertech, Eindhoven, 2015, pp. $1-6$.

[2] M. E. Baran and F. F. Wu, "Optimal capacitor placement on radial distribution systems." IEEE Trans. Power Del., vol. 4, no. 1, pp. 725734, 1989.

[3] C. Coffrin, H. L. Hijazi, and P. Van Hentenryck, "DistFlow extensions for AC transmission systems," [Math.OC], pp. 1-19, 2015.

[4] S. H. Low, "Convex relaxation of optimal power flow - part I: formulations and equivalence," IEEE Trans. Control Netw. Syst., vol. 1, no. 1, pp. 15-27, mar 2014.

[5] E. Dall' Anese, G. B. Giannakis, and B. F. Wollenberg, "Optimization of unbalanced power distribution networks via semidefinite relaxation," in North American Power Symp., Champaign, IL, USA, 2012, pp. 1-6.

[6] L. Gan and S. H. Low, "Convex relaxations and linear approximation for optimal power flow in multiphase radial networks," in Power Syst. Comp. Conf., Wroclaw, Poland, 2014, pp. 1-9.

[7] L. Gan, N. Li, U. Topcu, and S. Low, "On the exactness of convex relaxation for optimal power flow in tree networks," in IEEE Conf. Decision Control, 2012, pp. 465-471.

[8] F. Geth and C. Coffrin, "Direct method to recover current and voltage in multi-conductor optimal power flow models," in IEEE PES General Meeting, Atlanta, Georgia, 2019, pp. 1-5.

[9] I. Dunning, J. Huchette, and M. Lubin, "JuMP: a modeling language for mathematical optimization,” SIAM Review, vol. 59, no. 2, pp. 295-320, 2017.

[10] A. Wächter and L. T. Biegler, "On the implementation of primaldual interior point filter line search algorithm for large-scale nonlinear programming," Math. Prog., vol. 106, no. 1, pp. 25-57, 2006.

[11] B. Mahdad, T. Bouktir, and K. Srairi, "A three-phase power flow modelization: a tool for optimal location and control of FACTS devices in unbalanced power systems," in IEEE IECON, 2006, pp. 2238-2243.

[12] S. Kim, M. Kojima, and M. Yamashita, "Second order cone programming relaxation of a positive semidefinite constraint," Optimization Methods Software, vol. 18, no. 5, pp. 535-541, 2003.

[13] B. A. Mather, L. R. de Araujo, S. Paudyal, T. E. McDermott, J. C. Fuller, H. Zhu, W. Kersting, B. C. Pal, G. J. Shirek, L. F. Ochoa, J. L. R. Pereira, R. C. Dugan, C.-W. Ten, K. P. Schneider, and S. Matthias, "Analytic considerations and design basis for the IEEE distribution test feeders," IEEE Trans. Power Syst., vol. 33, no. 3, pp. 3181-3188, 2017.

[14] B. Kocuk, S. S. Dey, and X. A. Sun, "Matrix minor reformulation and SOCP-based spatial branch-and-cut method for the AC optimal power flow problem," 2017. [Online]. Available: https://arxiv.org/pdf/1703.03050.pdf

[15] H. Hijazi, C. Coffrin, and P. Van Hentenryck, "Polynomial SDP cuts for optimal power flow," in PSCC, Genoa, Italy, 2016.

[16] M. Fazel, H. Hindi, and S. Boyd, "A rank minimization heuristic with application to minimum order system approximation," Proc. American Control Conf., vol. 6, no. 2, pp. 4734-4739, 2001. [Online]. Available: http://ieeexplore.ieee.org/lpdocs/epic03/wrapper.htm?arnumber=945730 